\title{
LA MIGRACIÓN INTERNACIONAL EN LOS CENSOS DE 2010 EN LOS PAÍSES DE AMÉRICA LATINA Y EL CARIBE
}

\author{
Jorge Martínez Pizarro* \\ Laura Calvelo**
}

\begin{abstract}
El artículo aborda la medición de la migración internacional en los países de América Latina y el Caribe hacia 2010. Se ofrece un panorama a partir del análisis de diez censos de población que a mediados de 2012 habían cumplido su etapa de levantamiento. Se describen las experiencias censales tanto en sus fortalezas como en sus limitaciones, comparándolas con las prácticas precedentes, las recomendaciones internacionales y las modalidades adoptadas. Se menciona la emergencia de nuevos asuntos de interés, como las indagaciones por los emigrados.

Palabras clave: Medición de la migración internacional; Países de América Latina y el Caribe; Censos de población 2010.
\end{abstract}

\section{Introducción}

Promediando el período de levantamiento de la ronda de censos de 2010, este trabajo analiza brevemente el panorama de la medición de la migración internacional en los países de América Latina y el Caribe. Para ello, describe las experiencias censales existentes, tanto en sus fortalezas como sus limitaciones. Mencionamos por ejemplo, la emergencia de nuevos asuntos de interés que han ido surgiendo, y cuya medición puede ser crucial para muchos países, como las indagaciones por los emigrados. Tal como venimos destacando desde hace más de una década, ${ }^{1}$ en América Latina los censos de población

* Geógrafo y demógrafo, investigador del Centro Latinoamericano y Caribeño de Demografía (CELADE) - División de Población de la CEPAL. E-mail: jorge.martinez@cepal.org. Santiago del Chile/Chile.

** Demógrafa (Universidad Nacional de Luján) y Socióloga (Universidad de Buenos Aires), actualmente se desempeña como docente investigadora en la Facultad de Ciencias Sociales - Universidad de Buenos Aires (UBA). E-mail: lauracalv@sociales.uba.ar. Buenos Aires/Argentina.

1 MARTÍNEZ PIZARRO, Jorge. "La migración internacional en los censos de población", p. 61-90; IDEM. Desafíos para mejorar la información sobre migración internacional: notas de discusión; 
han constituido uno de los más importantes sustentos empíricos para el estudio de la migración internacional, desde las estimaciones sobre su cuantía hasta los patrones espaciales y las características sociodemográficas y socioeconómicas de las personas y los hogares migrantes. La información censal posibilitó la creación del Programa IMILA del CELADE, que comenzó en la década de 1970 y que ha sido, hasta la fecha, un proyecto modelo para otras regiones y base fundamental en la creación de sistemas de información migratoria.

Los censos siguen siendo la fuente matricial de información migratoria para los países de la región. Esta situación ocurre, en general, porque no se han desarrollado suficientemente alternativas como las encuestas, ni se han mejorado sustancialmente los registros administrativos. De ahí el interés por mostrar un adelanto de la experiencia habida en la ronda de 2010, según la información disponible a mediados de 2012, comparándola con las prácticas precedentes, las recomendaciones internacionales y las modalidades adoptadas.

Insistimos en que la información censal adolece de restricciones, pues los datos sobre el país de nacimiento se refieren solo a los stocks acumulados de migrantes y no estrictamente a los flujos. ${ }^{2}$ Además, dada la naturaleza periódica con que se realizan, los censos entregan datos que, en algunas dimensiones, pueden perder vigencia al transcurrir unos años, con la particularidad de seguir leyéndose "actuales" después de mucho tiempo - un típico ejemplo es la cuantía de los inmigrantes de algún origen específico. Las restricciones propias de las operaciones censales obligan a pensar en los límites que ofrecen $y$, en ese contexto, en la necesidad de emplear apropiadamente fuentes alternativas, en especial teniendo en cuenta el exacto alcance de la información que generan (stocks, en general una versión restringida de flujos, y la distinción de algunos tipos de migrantes).

\section{La experiencia acumulada en la medición de la migración internacional en los censos de población en la región}

Los países de la región latinoamericana y caribeña cuentan con una tradición de captación y estimación de la migración internacional centrada en los censos de población. Estos han provisto tradicionalmente el sustento empírico para su estudio, tanto desde el punto de vista de las estimaciones sobre su cuantía como del análisis de los patrones espaciales y las características sociodemográficas y socioeconómicas de los migrantes. ${ }^{3}$

\footnotetext{
IDEM. “Medición e información sobre la migración internacional a partir de los censos: lecciones, desafíos y oportunidades", p. 97-134.

2 IDEM. "La migración internacional..., op. cit.

IDEM. "Medición e información..., op. cit.
} 
La información censal se releva en forma universal y simultánea y permite calcular el stock de inmigrantes nacidos en el extranjero, estudiar las características de la población inmigrante, conocer los cambios en la composición de los inmigrantes por período de llegada e identificar la incidencia del retorno de nativos. Desde el punto de vista de la provisión de insumos demográficos, los censos registran datos de stocks, es decir, de una cifra acumulada de inmigrantes, pero producen la información necesaria para estimar saldos migratorios de los períodos intercensales mediante la aplicación de métodos indirectos. ${ }^{4}$

Adicionalmente, con los datos de un censo de población se pueden realizar análisis comparativos de los atributos de la población migrante respecto de la no migrante a los niveles de desagregación geográfica deseados y obtener estimaciones sobre los hogares con población nacida en otros países.

Los censos también brindan una aproximación al conocimiento de la emigración de los nativos. Al intercambiar los datos de los stocks, se obtienen estimaciones de los emigrantes, cuya cobertura dependerá del número de países de destino representados en el intercambio. Además, el censo del país de origen permite incluir preguntas específicas para estimar los hijos sobrevivientes y los ex-miembros de los hogares residentes en el exterior (si bien esta aproximación adolece de sesgos difíciles de cuantificar). Al mismo tiempo, sus datos proveen insumos para la aplicación de métodos indirectos de estimación de saldos migratorios. Lo más relevante de los censos cuando se consideran los datos de los países de destino es que informan sobre los stocks acumulados y permiten conocer las principales características sociodemográficas mediante la información sobre el país de nacimiento y el año o fecha de llegada.

Las principales variables relevadas por los censos de población son el país de nacimiento, el lugar de residencia fecha fija anterior - usualmente cinco años antes del censo, es decir a mitad del período intercensal -, el lugar de residencia habitual en el momento del censo $^{5}$, el año de llegada y la nacionalidad. Se encuentran también todas las características sociales y demográficas de los inmigrantes, de sus hogares y las características de las viviendas que habitan.

El seguimiento de las variables referidas a la migración internacional incluidas por cada país en los censos de las rondas de 1980 hasta 2000 demostró la conformación de un núcleo de variables básicas común a todos

${ }^{4}$ CALVELO, Laura. "Viejos y nuevos asuntos en las estimaciones de la migración internacional en América Latina y El Caribe".

5 Sólo en la metodología de los censos de hecho. 
los países, la unificación de criterios en torno de la definición de los migrantes internacionales y el reconocimiento de temáticas emergentes comunes a la mayor parte de los países. En la base de esa práctica de estimación a partir de los censos de población se encuentra la definición tradicional de migración internacional entendida como el traslado de residencia de las personas de un país a otro. La ronda de censos de 2000 ya había demostrado la consolidación de la noción tradicional para captar el stock de inmigrantes (nacidos en el exterior) e incluso su extensión - entonces a diez países ${ }^{6}$ - por medio de la incorporación de nuevas variables para captar individuos identificados como ex-miembros de los hogares residiendo en el exterior. ${ }^{7}$

Sin embargo, las estimaciones del stock de inmigrantes y la aproximación al acervo de ex -miembros del hogar residentes en el exterior constituyen una parte de la información procesada. Junto a las cuantías aparece la identificación de atributos sociodemográficos, económicos y espaciales que aportan la mayor riqueza de la fuente censal. ${ }^{8}$ Dicha información ha demostrado ser muy útil en la elaboración de perfiles, abriendo importantes posibilidades para el conocimiento de disimilitudes entre los atributos de la población migrante y no migrante, nativa y nacida en el extranjero, y para profundizar el estudio de subpoblaciones específicas (determinadas colectividades o grupos de sobrevivientes de cohortes migratorias).

Debe reiterarse que el concepto consolidado de migración, esto es, el traslado del país de residencia, origina información susceptible de intercambiarse entre los países para el abastecimiento y la actualización de la matriz migratoria regional. Tal es el principio fundante del Programa IMILA, Investigación de la Migración Internacional en Latinoamérica (CELADECEPAL), a partir del cual es posible estimar stocks de emigrantes (y sus especificidades) en los países incluidos en la matriz. ${ }^{9}$

La experiencia de las rondas de 1980, 1990 y 2000 demostró que las preguntas sobre el país de nacimiento y el de residencia cinco años atrás habían sido empleadas en la mayor parte de los países, particularmente en forma universal en cuanto al país de nacimiento. ${ }^{10}$ Algo similar ocurría con el año o fecha de llegada al país de residencia actual, que había

\footnotetext{
${ }^{6}$ Se trató de Colombia, Ecuador, El Salvador, Guatemala, Honduras, México, Nicaragua, Panamá, Perú y República Dominicana.

MARTÍNEZ PIZARRO, “Medición e información..., op. cit.

8 Ibidem.

9 Para profundizar en las características de IMILA y los países incluidos puede consultarse MARTíNEZ PIZARRO, Desafíos para mejorar..., op. cit.; IDEM, “Medición e información..., op. cit.

${ }^{10}$ Se trata del país de nacimiento del individuo o, como aconteció en la ronda de 2000, el de residencia de la madre al nacer.
} 
incrementado su presencia en las boletas de la ronda de 2000 respecto de 1990. Una interrogante adicional, que resulta fundamental para cotejar la residencia anterior con la del momento del empadronamiento, es la del país de residencia habitual, que tampoco ha sido generalizada, en virtud de su pertinencia más bien en los censos de hecho. Preguntas como la nacionalidad, la duración de la residencia y el país de residencia anterior siguieron siendo menos frecuentes. ${ }^{11}$

El uso del país de nacimiento y del país de residencia cinco años antes dio sustento a la identificación de los migrantes recientes (aquellos arribados en el último quinquenio) y los antiguos (que residían con antelación), los que en conjunto suman el stock acumulado de inmigrantes nacidos en el exterior sobrevivientes al momento del censo. Los nativos retornados son captados como parte de la migración internacional de retorno del período (es decir, nativos del país de empadronamiento que hace 5 años residían en el extranjero y que en el momento del censo residen en el país de empadronamiento).

La captación de la fecha de llegada de los nacidos en el exterior se generalizó en la ronda de 2000. La carencia de un período de referencia que deriva de la pregunta sobre el país de nacimiento limita el análisis del stock de migrantes acumulados. Como solución, se siguió empleando la distinción del año o fecha en que la persona que nació en otro país llegó a aquel en que reside actualmente. Las preguntas pertinentes, recomendadas por el CELADE y la Unión Internacional para el Estudio Científico de la Población (UIECP), comenzaron a formularse en los censos de 1980 y 1990 en algunos países, y hacia la ronda de 2000 prácticamente todos preguntaron por la fecha de llegada al país.

La pregunta referida a la nacionalidad ha sido incorporada en pocos países de la región. En la ronda de 2000 sólo tres la contemplaron en sus boletas censales, a pesar haberse recomendado el registro del país de nacimiento y del "país de ciudadanía". ${ }^{12}$

\section{Las recomendaciones para la ronda de Censos $\mathbf{2 0 1 0}$}

En el contexto previo a la ronda de censos de 2010, las recomendaciones internacionales en materia de producción de datos estadísticos y los acuerdos regionales referidos puntualmente a los censos de población habían señalado el conjunto mínimo de arreglos a mantener para asegurar el relevamiento de

\footnotetext{
11 Ibidem.

12 UNITED NATIONS. Principles and Recommendations for Population and Housing Censuses.
} 
información migratoria armonizada y en condiciones de ser intercambiada entre los países. ${ }^{13}$

Tal como oportunamente se sistematizara en las instancias supranacionales se ha ratificado que en los países de América Latina y el Caribe se reconoce al censo de población como pilar de las estimaciones de la migración internacional. ${ }^{14}$ Los censos de población reúnen las preguntas básicas cuyo comportamiento es conocido, correcto y garantiza "un mínimo posible" en las estimaciones y en el conocimiento migratorio.

Las tres rondas censales precedentes enseñan que la estimación de los stocks derivada de la pregunta sobre el país de nacimiento, así como la aproximación al conocimiento de ciertos flujos mediante el país de residencia cinco años antes, han sido de mucha utilidad. Similar reconocimiento merece la pregunta sobre el año o fecha de llegada, en tanto que la duración de la residencia, el país de residencia anterior y los hijos sobrevivientes en el exterior han demostrado ser indagaciones poco frecuentes. La identificación del país de nacimiento y de residencia cinco años antes no presenta dificultades, en tanto que persisten errores significativos en la captación del año o fecha de llegada según se formule la pregunta. Por otra parte, en los censos de hecho es necesaria la inclusión del país de residencia habitual, para la estimación de la migración de período.

La experiencia común a todos los países en las tres últimas décadas censales se expresa en el alto grado de homogeneización de las preguntas sobre migración internacional, lo que redunda en la minimización de defectos en la calidad de las respuestas, en las facilidades de procesamiento y en las posibilidades de intercambio de la información.

Al mismo tiempo, dadas su universalidad y robustez, los censos de población han ofrecido la posibilidad de explorar la captación de temáticas novedosas, llevando al límite las posibilidades que ofrecen sus datos. Sin embargo, el censo no es una fuente apropiada para el abordaje de temáticas excesivamente complejas o "sensibles", tales como la irregularidad migratoria, la situación de los solicitantes de refugio, las motivaciones para migrar o los ingresos percibidos por las remesas.

Lo relevante es que la mantención de criterios comunes permitió la producción de información susceptible de ser intercambiada entre los países, por medio de la provisión de insumos para el banco de datos del IMILA.

\footnotetext{
${ }^{13}$ Ibidem.

${ }^{14}$ CALVELO, op. cit.
} 
La aplicación de técnicas indirectas para estimar la emigración, como la referida a los hijos sobrevivientes residiendo en el exterior, no se han generalizado entre los países, en tanto que en los censos de la década de 2000 se evidenció el interés por conocer la presencia de ex-miembros del hogar residiendo en el exterior y la recepción de remesas.

Acerca de los contenidos básicos se había recomendado mantener el relevamiento del país de nacimiento, del país de residencia cinco años antes y de la fecha de llegada al país (o año de llegada en que se fijó la residencia actual en el país), consideradas como las tres preguntas básicas de medición de la migración internacional. En referencia a la captación del año de llegada se indicó incluir eventualmente el relevamiento del período de llegada como alternativa de respuesta para los casos en que falla la captación del año de llegada. Cabe destacar que esta consulta por la fecha de llegada, en particular el último arribo, permite conocer el tiempo de residencia de los inmigrantes (una aproximación a los flujos), y se ha sugerido no interrogar directamente por el lapso de tiempo que lleva alguien en el país, porque de ese modo la información perdería precisión. Contrariamente se recomendó registrar el mes y año de llegada, y tabular como "período de llegada". ${ }^{15}$ Considérese que en la ronda 2000 los niveles de no respuesta del año de llegada permanecieron en niveles muy altos. En la mayor parte de los casos una altísima no respuesta impide utilizar la información proporcionada por la variable, situación que ejemplificaron Ecuador, Paraguay, Honduras y Bolivia, un grupo de países con no respuesta superior al 20 por ciento. En otros una ausencia de información más moderada obliga a tomar los datos con reserva: Argentina, Chile, República Bolivariana de Venezuela y Costa Rica, todos con valores entre 15 y 20 por ciento.

En los censos de hecho se puntualizó incluir la captación del país de residencia habitual y considerar que la información sobre nacionalidad no reemplaza la pregunta sobre el país de nacimiento para el estudio de la migración internacional.

Respecto de la captación y tratamiento de la información se recomendó no restringir la aplicación de las preguntas básicas de la migración a muestras de la población cuando se realizan censos con muestreo ${ }^{16}$, y codificar la información de países siguiendo normas internacionales, garantizando su comparabilidad y la identificación de todos los países declarados por la población.

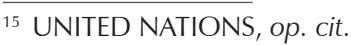

${ }^{16}$ El CELADE recomienda permanentemente la inclusión de todas las preguntas sobre migración en los formularios básicos (CELADE. Censos de población de 1990: selección de documentos del CELADE). 
Para incluir la captación de la nacionalidad se instó a proceder de acuerdo a las recomendaciones internacionales de las Naciones Unidas, que puntualizan definir la pregunta por el país de nacionalidad y no por la nacionalidad a secas. ${ }^{17}$ En la perspectiva de la estimación de la migración internacional es evidente, además, que la información sobre la nacionalidad legal requiere, de todos modos, el empleo simultáneo de la pregunta sobre el país de nacimiento. ${ }^{18}$

Finalmente se recomendó no probar la captación de nuevas temáticas de la migración internacional sin la previa exploración de sus fortalezas y debilidades y si no son explotados debidamente los datos de carácter tradicional.

\section{La medición de la migración internacional en los Censos de $\mathbf{2 0 1 0}$}

Al $1^{\circ}$ de julio de 2012, la ronda de censos de 2010 en la región latinoamericana y caribeña se encontraba promediando su levantamiento. Hasta esa fecha, diez países habían concluido la etapa de relevamiento de sus censos de población: Argentina, Brasil, Costa Rica, Chile, Ecuador, México, Panamá, República Dominicana, Uruguay y República Bolivariana de Venezuela. ${ }^{19}$

El análisis de los contenidos incluidos en el diseño conceptual y operacional de las boletas de los censos levantados debe ser tomado como una aproximación válida y razonable - aunque provisoria - al panorama de la medición de la migración internacional en la ronda de 2010 (véanse los cuadros 1 y 2). Si bien entre los países que se analizan se encuentra cerca del 75 por ciento de la población de América Latina y el Caribe (de acuerdo a las previsiones demográficas vigentes, CEPAL-CELADE, 2009), las conclusiones ofrecidas deberán ser confirmadas con el análisis de los censos pendientes.

Hasta el momento, puede afirmarse que la captación de la migración internacional en la ronda de censos de 2010 no ha registrado variaciones sustantivas respecto de la ronda de 2000. Al respecto aparecen tres constataciones principales. En primer lugar, continúan vigentes el concepto y la operacionalización de la migración internacional de rondas anteriores. En segundo término, se consolida la captación de información referida a la

\footnotetext{
17 UNITED NATIONS, op. cit.

${ }^{18}$ En rigor, se recomienda recoger información que permita clasificar a la población en: a) ciudadanos por nacimiento; b) ciudadanos nacionalizados por distintas razones, y c) extranjeros. Se sugiere no usar un adjetivo para indicar la ciudadanía, sino registrar el país de ciudadanía como tal (ibidem).

${ }^{19}$ Ocho países a mediados de 2012 han iniciado la difusión de resultados, si bien con distintos grados de avance: Argentina, Brasil, Costa Rica, Ecuador, México, Panamá, República Dominicana y Uruguay.
} 
emigración internacional de latinoamericanos. Finalmente, se extiende la metodología de los censos de derecho, mejorando el conocimiento del país de residencia habitual, cuestión que en los censos de hecho depende de la correcta captación de esa variable.

Se mantiene plenamente vigente la definición tradicional de migración internacional como traslado de la residencia de las personas de un país a otro, identificándose el traslado respecto de una residencia anterior (el país en que la persona nació y aquel en que vivía hace cinco años) y captándose un solo movimiento de tipo directo. En la base de estas operaciones continúa la consideración de la habitualidad de la residencia actual como el país donde estaba ubicado el lugar en que se residió durante los últimos seis meses. ${ }^{20}$

El diagnóstico de la migración internacional proporcionado por estos nuevos censos de población incluye el relevamiento de información relativa tanto a la inmigración internacional como a la emigración que tiene lugar en los países de América Latina y el Caribe, con significativas salvedades en casos puntuales. Tal como se venía viendo en las rondas de 1990 y 2000, se afianza la inclusión de preguntas relativas a la existencia de ex-miembros del hogar residiendo en el exterior, cuestión relevante dado que las fuentes y sistemas de información de la región (básicamente IMILA) no recaban datos referidos a países de destino extrarregional. De tal forma los censos siguen contabilizando inmigrantes internacionales (tanto nacidos en el extranjero como nativos) y ex-miembros de los hogares residiendo en el exterior. Esta última indagación, en nuestra opinión, no se ha probado consistente, aunque reviste potencialidades analíticas. De cualquier forma, se confirma el consenso generalizado acerca de la gravitación adquirida por la emigración extrarregional de latinoamericanos y caribeños y por la preferencia de los institutos de estadística de su relevamiento mediante técnicas de fácil aplicación, aunque de menor precisión, para proveer insumos a estimaciones ajustadas del cuantum de la emigración. Los métodos de estimación indirecta de naturaleza demográfica, tales como los métodos de Somoza - de hijos sobrevivientes residiendo en el exterior - y Hill -de residencia de los hermanos - implican el cumplimiento efectivo de una serie de supuestos, requieren para su aplicación de otros insumos del análisis demográfico y demostraron muy escasa presencia en la ronda de 2000, cuando sólo Haití incluyó las preguntas requeridas por el método de Somoza. ${ }^{21}$

\footnotetext{
${ }^{20}$ El período definitorio puede registrar variaciones entre países.

${ }^{21}$ En la ronda de 1980 cuatro países incluyeron las preguntas requeridas por el Método (Colombia, Paraguay; República Dominicana y Uruguay); en la de 1990 lo hicieron tres países (Bolivia, Colombia y República Dominicana); y en la de 2000 sólo uno (Haití).
} 
En tercer lugar, se sigue extendiendo la metodología de los censos de derecho en los países de la región, lo que a los fines del estudio migratorio conlleva la ventaja de proveer desde el empadronamiento mismo de la población su distribución por lugar (país) de residencia habitual, pudiendo prescindirse de la pregunta en el cuestionario. Siete de los diez países analizados han realizado censos de derecho en la ronda de 2010 (Brasil, Costa Rica, Chile, México, República Dominicana, Uruguay y República Bolivariana de Venezuela) y tres mantienen la metodología de hecho (Argentina, Ecuador y Panamá). ${ }^{22}$ A pesar de dicho avance en la metodología censal que, entre otras ventajas, permite utilizar cuestionarios más largos durante un período de empadronamiento prolongado, hasta el momento no parece significativa la cantidad de países que incluyen preguntas sobre el país de ubicación del trabajo principal (para la población ocupada), o sobre el país de estudio de la población que se encuentra estudiando. Tales indagaciones, relativas al conocimiento de otras formas de movilidad espacial, reconocen antecedentes en los censos de algunos países desde la ronda de $1980 .{ }^{23}$

Una última observación general se refiere a la presencia del muestreo en los operativos censales. En la ronda de 2010, de los diez censos levantados, tres han puesto en práctica el relevamiento por muestreo en las localidades urbanas de mayor tamaño. A pesar de las recomendaciones que indicaban la inclusión de las variables de la migración en los cuestionarios básicos, Argentina, Brasil y México las ubicaron en sus cuestionarios ampliados que se aplican por muestreo. Para valorizar tal decisión en los tres países considérese que hacia 2010 según las proyecciones vigentes ${ }^{24}$ Argentina, Brasil y México reúnen alrededor del 60 por ciento de la población regional.

El país de nacimiento, variable principal del estudio migratorio en la región, ha sido relevado por todos los países. La mayor parte de ellos preguntó por el país de nacimiento de los individuos, en tanto que tres países desde la ronda de 2000 han mantenido la operacionalización por medio de la identificación del país de residencia de la madre al momento del nacimiento (Costa Rica, Chile y Panamá).

El país de residencia 5 años atrás se contempló en ocho de los diez países analizados: Brasil, Costa Rica, Chile, Ecuador, México, República Dominicana, Uruguay y República Bolivariana de Venezuela. En tanto

\footnotetext{
${ }^{22}$ En la ronda de censos de 2000 trece países de la región efectuaron censos de derecho: Brasil (2000), Cuba (2002), Colombia (2005-2006), Costa Rica (2000), El Salvador (2007), Guatemala (2002), Haití (2001), Honduras (2003), México (2000), Nicaragua (2005), República Dominicana (2002), Uruguay (Censo 2004 - Fase I) y Venezuela (2001).

${ }^{23}$ MARTÍNEZ PIZARRO, "Medición e información..., op. cit.

${ }^{24}$ CEPAL-CELADE. Observatorio Demográfico 7 Proyección de población.
} 
Panamá no lo incluyó (tampoco lo había incluido en la ronda de 2000), y Argentina relevó el lugar de residencia 5 años antes sin especificar el país (cuando el lugar estaba ubicado en "Otro país").

El año de llegada presenta un panorama favorable en la mayor parte de los cuestionarios analizados, tomando en cuenta la complejidad de la variable y sus históricos problemas de calidad en el marco de la fuente censal. En su versión recomendada de relevamiento, es decir como fecha o año de llegada - idealmente la última Ilegada -, fue aplicada por ocho países (Brasil, Costa Rica, Chile, Ecuador, México, República Dominicana, Uruguay y República Bolivariana de Venezuela). Argentina y Panamá optaron por registrar sólo el período de llegada, lo que restaría precisión a la medición al suprimir las posibilidades de intercambio de datos compatibles y armonizados entre países. ${ }^{25}$

El país de residencia anterior fue contemplado en tres censos (de Brasil, Panamá y Uruguay), lo que constituye una presencia atenuada de la pregunta que en el caso de Brasil y Panamá había sido aplicada en la ronda de 2000. En tanto hasta el momento, la duración de la residencia aparece en la ronda de 2010 como una indagación exclusiva de Uruguay.

La captación de la nacionalidad presenta una situación diversa. Mientras en la ronda de 2000 sólo tres de los veinte países la había introducido, en la ronda de 2010, de los diez países con información, tres la han contemplado: Brasil (que ya la incluía en 2000), Chile y Ecuador (que la incorporan recientemente). Costa Rica dejó de preguntarla en 2010 luego de contar con ella en 1980 y 2000. En la República Bolivariana de Venezuela no se incluyó la pregunta luego de utilizarla en 1990 y 2000. Debe recordarse que en la región sólo Brasil mantiene la pregunta de la nacionalidad a lo largo de las últimas cuatro rondas censales, lo que constituye una constante significativa marcada por el IBGE en estricto cumplimiento, por otra parte, del contenido de la recomendación internacional que prescribe identificar ciudadanos por nacimiento, ciudadanos nacionalizados por distintas razones, y extranjeros. ${ }^{26}$

México, país destacado por la cantidad, variedad y robustez de sus fuentes estadísticas para el seguimiento de la migración internacional, se diferencia en la ronda de 2010 al prescindir del país de residencia 5 años antes y al conservar una pregunta que sólo tiene antecedente censal en la ronda 2000 del país, la pregunta sobre motivos de la inmigración.

\footnotetext{
${ }^{25}$ Los períodos de llegada definidos en la pregunta de Argentina son “Antes de 1991; 1991 a 2001; 2002 al momento", en tanto que Panamá aplicó "Antes de mayo de 2000; mayo 2000 a abril 2005; y mayo 2005 al momento de empadronamiento".

${ }^{26}$ UNITED NATIONS, op. cit.
} 


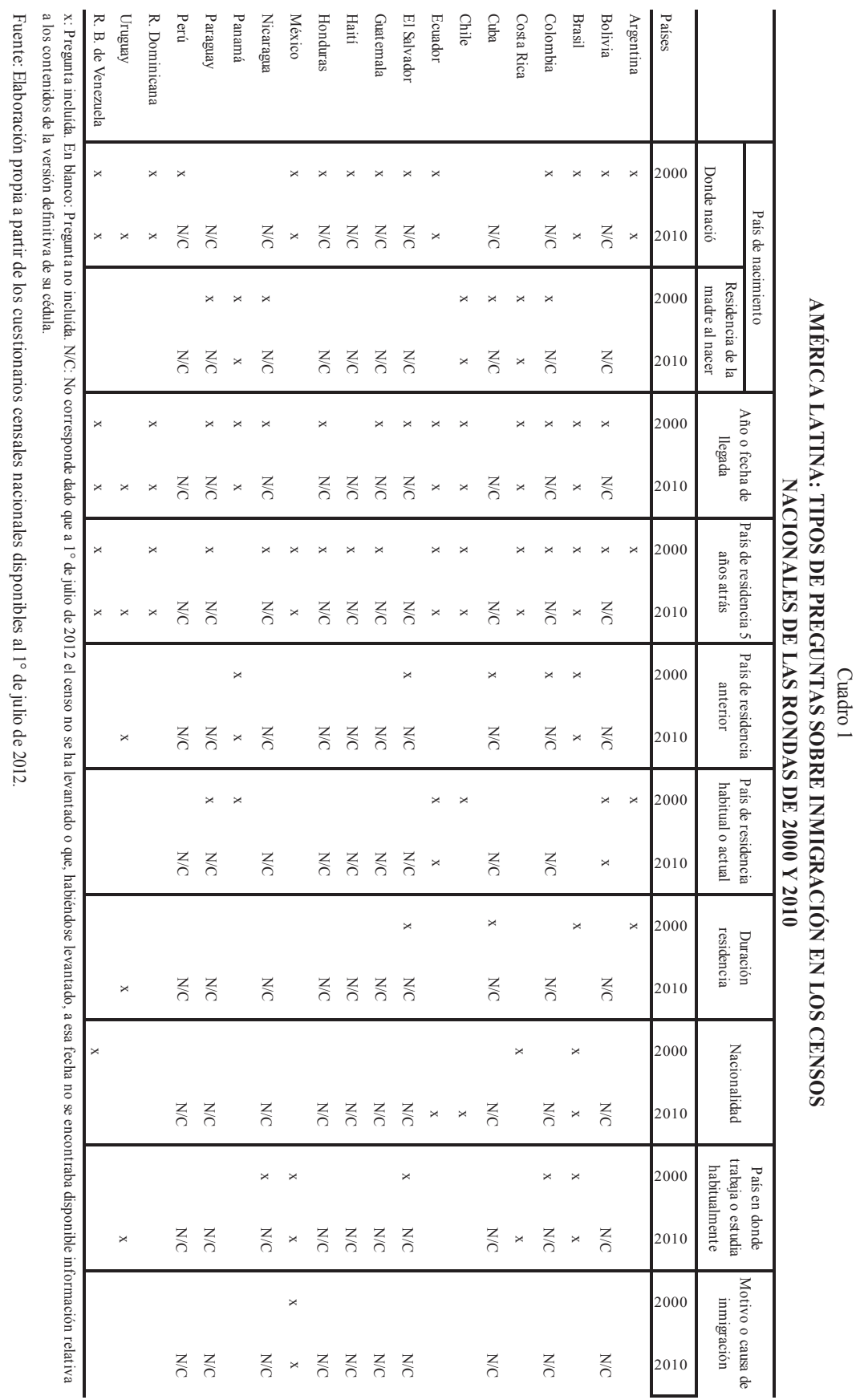


Por último, el relevamiento de atributos ligados a la existencia de otras expresiones del concepto de movilidad en el marco de los censos de población, presenta desarrollo en los censos de Brasil, Costa Rica, México y Uruguay, quedando los seis países restantes sin haber considerado la temática. Brasil y México han demostrado continuidad en la captación entre las rondas de 2000 y 2010. Se trata de la búsqueda de indicadores apropiados de la movilidad cotidiana de alcance internacional, que parece restringirse en la ronda de 2010 a la dimensión económica, al relevarse en todos los casos como característica de la ocupación principal de la población, preguntándose por la ubicación del lugar de trabajo. No obstante, deberá esperarse a los demás censos de la ronda para concluir este panorama.

La captación de ex-miembros del hogar en el exterior constituye una opción en los censos de la región impuesta, probablemente, por la necesidad de producir información relativa a la emigración extrarregional en los países de América Latina, en un contexto de ausencia de fuentes de datos locales y de escasez de evidencia empírica en los países de destino. ${ }^{27}$ No debe olvidarse que hasta la ronda de censos 2000 los países de Europa no difundían información de la población empadronada por país de nacimiento sino por nacionalidad; y que significativas cuotas de latinoamericanos permanecían invisibilizadas entre los ciudadanos europeos por haber recuperado la nacionalidad de sus antepasados. Recién a partir de la intensa inmigración internacional recibida por algunos países de Europa se generalizó la divulgación del país de nacimiento. ${ }^{28}$

La consulta específica adquiere relevancia en la medida que IMILA no rescata información de países de destino como los europeos, que tienen importantes contingentes de emigrantes de la región. La pregunta más frecuente ha sido aquella destinada a identificar si algún ex-miembro del hogar se ha ido a vivir a otro país, interrogando por ciertas características sociodemográficas de cada "emigrante" (sexo, edad al partir, edad actual, país de destino, país de residencia actual, año o período de salida, año de retorno, nivel educativo y/o causa de la partida, dependiendo de cada caso). Sin embargo, se ha insistido en que no se trata de una estimación de la emigración y que no cabe interpretar este resultado como magnitud de emigrados, advirtiéndose sobre diversas limitaciones que aconsejan manejra con mucha cautela la información obtenida. ${ }^{29}$

${ }^{27}$ CALVELO, op. cit.

${ }^{28}$ IDEM. "La emigración argentina y su tratamiento público (1960-2003)"; IDEM. "La emigración en Argentina: 1960-2000".

${ }^{29}$ MARTíNEZ PIZARRO, "Medición e información..., op. cit.; MAGUID, Alicia. "La emigración internacional a través de los censos en países de origen: evaluación de resultados y recomendaciones". 
El abordaje de la emigración en los censos de América Latina y el Caribe se extendió considerablemente en la década de 2000, cuando diez países incluyeron la captación de ex-miembros del hogar viviendo en el exterior (Colombia, el Ecuador, El Salvador, Guatemala, Honduras, México, Nicaragua, Panamá, Perú y la República Dominicana), en tanto que en la ronda de 1990 sólo tres habían experimentado ese abordaje (El Salvador, Guatemala y Panamá).

Hasta el momento en la ronda de 2010 de los diez países analizados cinco incluyeron la temática en sus boletas censales: Brasil, Costa Rica, Ecuador, México y Panamá. De ellos, Ecuador, México y Panamá dieron continuidad a una indagación ya presente en sus boletas desde la ronda de 2000, en tanto que en el caso de Brasil y Costa Rica se introduce por primera vez el bloque de preguntas.

Dos comentarios generales deben realizarse. Por un lado aparecen países que permanecen desprovistos de alternativas censales que consideren la emigración extrarregional (Argentina, Chile, República Dominicana, Uruguay y República Bolivariana de Venezuela), lo que revela distintos escenarios en materia de fuentes de datos, diferentes realidades migratorias y discrepancias en sus valorizaciones por parte de los institutos de estadística. En el caso de Argentina, la emigración permanece ausente en los censos y las encuestas de hogares, en tanto que en Uruguay la emigración no se mide en los censos, pero presenta antecedentes en la Encuesta Anual de Hogares.

En otro extremo se encuentran los países que incluyen la temática en sus censos, conformando una amplia variedad de abordajes operacionales de la metodología de captación de ex-miembros del hogar en el exterior. Tal como se observó en la ronda 2000, los períodos y momentos de referencia de la pregunta principal en la ronda de 2010 presentan significativas discrepancias. Brasil realiza la pregunta en referencia a ex-miembros que vivían en el exterior a una fecha fija (mitad del año 2010). México lo hace en relación a personas que habiendo formado parte del hogar partieron a vivir a otro país durante los cinco años anteriores al censo; y Panamá lo hace refiriéndose a los diez últimos años. Costa Rica pregunta acerca de personas que formaban parte del hogar y se encuentran viviendo en el exterior al momento del censo (sin momento de referencia preciso). Y Ecuador consulta sobre personas que "desde el censo anterior (noviembre de 2001) viajaron a otro país y todavía no regresan para quedarse definitivamente". 


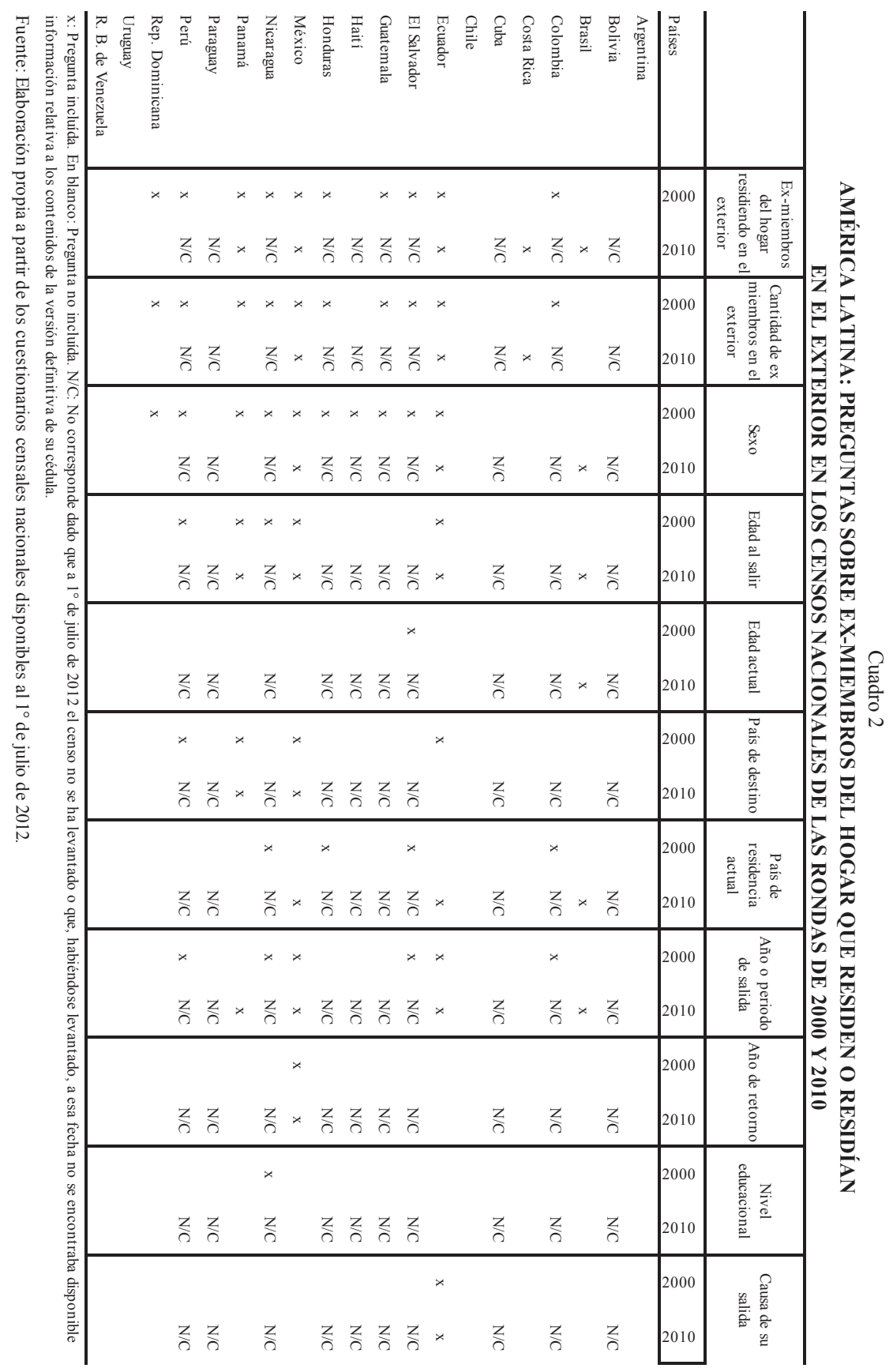


Partiendo de tales disimilitudes para definir el universo de ex-miembros a ser contabilizados, la inclusión de características sociodemográficas es igualmente diversa. La cantidad de ex-miembros es registrada por Costa Rica, Ecuador y México; el sexo es incluido por Brasil, Ecuador y México; la edad es incluida por Brasil como año de nacimiento, por Ecuador como edad al salir del país, por México como edad al salir del país por última vez y por Panamá como edad al partir.

Más homogénea ha sido la captación del año de partida y del país de residencia de los ex-miembros, incluidos por cuatro de países: Brasil, Ecuador, México y Panamá. En tanto, sólo México indagó sobre el lugar de última residencia en el país de empadronamiento - antes de partir - y de hecho es el país con mayor cantidad de variables descriptoras de los ex-miembros, lo que puede observarse en el cuadro 2, donde se han volcado todos los contenidos hasta aquí descriptos en comparación con los respectivos de la ronda de 2000.

Una última observación referida a la inclusión de la temática de las remesas. La identificación de hogares perceptores de remesas se encontraba entre los temas emergentes de la ronda de 2000, cuando cinco países incluyeron preguntas sobre su percepción (El Salvador, México, Nicaragua, Perú y República Dominicana). Hasta el momento en la ronda de 2012, de los diez países analizados tres incorporaron su medición: Costa Rica, Ecuador y México. Costa Rica captó a nivel de los hogares no sólo la percepción sino también el envío de remesas; en tanto Ecuador incluyó la percepción a nivel de los hogares; y México optó por considerar en su formulario ampliado la recepción de remesas a nivel de los individuos mayores de 12 años.

\section{Conclusión}

El avance de la ronda de censos de 2010 permite avizorar las primeras certezas sobre el panorama de la medición de la migración internacional en los países de América Latina y el Caribe. Ante el levantamiento de los diez primeros censos de la ronda resulta llamativa la homogeneidad prevaleciente en conceptos y definiciones en dos órdenes. Por un lado, como aspectos positivos, están la robustez de la definición operacional del migrante internacional, a través del uso de criterios definitorios tanto en sentido absoluto como de período. Por otro lado, en relación a la atención común de temáticas consideradas emergentes en las rondas anteriores, sobresale la captación de ex-miembros del hogar residiendo en el exterior, que parece ser valorizada por su carácter alternativo a la medición de la migración latinoamericana extrarregional, si bien habrá que evaluar la utilidad que presta. 
Simultáneamente aparecen heterogeneidades de procedimientos que demuestran que la región debe seguir trabajando en el mejoramiento, la armonización y la compatibilización de información migratoria. Persisten significativas discrepancias en las definiciones operacionales de numerosos sistemas clasificatorios, como lo ilustran el lugar de residencia 5 años antes (que no siempre requiere especificar el país) y la captación del año o fecha de llegada (que a pesar de los esfuerzos realizados en defensa de su precisión, no siempre se combinó con el período de llegada). Sin embargo, la mayor heterogeneidad se presenta en la operacionalización de los ex-miembros del hogar residiendo en el exterior, un boque de variables más recientes en el acervo de la medición de la migración en América Latina y el Caribe. Las discrepancias en los períodos de referencia aparecen como un obstáculo insalvable en materia de comparabilidad de la magnitud de ex-miembros captados. Por último, si bien aún no es posible concluir diagnósticos en la ronda de 2010, la detección de hogares que perciben remesas viene ocupando un espacio considerable, demostrando que sigue siendo una temática de interés en países con significativas cantidades de nativos en el exterior.

Paralelamente avanza la metodología de censos de derecho, lo que contribuye a una mejor determinación de la población residente habitual del país. En contrapartida, el uso del muestreo en áreas densamente pobladas sigue encontrando variables básicas de la medición migratoria en los cuestionarios ampliados, lo que desconoce la importancia de las recomendaciones internacionales e impone límites a las posibles desagregaciones de la información a escalas menores para universos de migrantes cuantitativamente escasos.

La medición de la migración internacional en los censos de población cobra una renovada relevancia a la luz del contexto internacional posterior a la crisis financiera iniciada en 2008 y sus consecuencias en el comercio mundial y en la economía de los países desarrollados hacia donde se dirigía el grueso de la migración latinoamericana extrarregional. Los movimientos internacionales de población forman parte de las redefiniciones en curso, están sometidos a las cambiantes condiciones de los mercados de trabajo de los países centrales y a la persistencia y endurecimiento de las restricciones al ingreso y la permanencia de inmigrantes extrarregionales.

La incertidumbre que se cierne sobre la vigencia de las tendencias migratorias recientes en América Latina y el Caribe refuerza la necesidad de unificar criterios de medición y de producir información estadística relativa a la migración internacional en condiciones de ser intercambiada para nutrir la matriz común de datos a nivel regional, el IMILA del CELADE. A nivel de 
los países parece claro que las nuevas demandas de información encontrarán mejor preparadas a las naciones dotadas de fuentes de datos válidos y oportunos, y de las variables básicas imprescindibles para cubrir la variedad de estimaciones necesarias a un diagnóstico migratorio íntegro. A modo de ejemplo, considérese la valuación del perjuicio de no disponer del país de residencia 5 años antes cuando se vuelva valioso el conocimiento del retorno de los nativos en el exterior. Tómese en cuenta, también, la imposibilidad de desagregar con suficiente precisión la fecha de llegada de la población nacida en el extranjero para identificar cohortes migratorias recientes en el marco de posibles redireccionamientos de los flujos migratorios ante la recesión mundial.

A nivel de la región en conjunto es evidente que la potencialidad analítica y empírica de la información sobre la migración internacional es un reto común. Las posibilidades de abastecer los requerimientos de los países parten de la disponibilidad de datos comparables por parte de cada uno de ellos. En el mantenimiento del sistema de información regional sustentado en los censos de población, se cifran las posibilidades de consolidar estimaciones de alcance latinoamericano y caribeño.

Por último, vale reiterar la importancia de los avances en materia de producción de datos y la vigencia de ese llamado en el marco de los acuciantes problemas económicos y sociales de los contextos donde se desenvuelven los migrantes de la región y donde se ven amenazados los principios de protección de sus derechos. ${ }^{30}$ La información, como parte fundamental de las acciones inspiradas en la defensa de los derechos humanos de los migrantes y sus familias, debe aparecer en el primer plano de la agenda de trabajo regional.

\section{Bibliografia}

CALVELO, Laura. "Viejos y nuevos asuntos en las estimaciones de la migración internacional en América Latina y El Caribe", in Série Población y Desarrollo, n. 98. CELADE - CEPAL. Santiago de Chile: Naciones Unidas, 2011.

. "La emigración argentina y su tratamiento público (1960-2003)", in III Congreso de la Asociación Latinoamericana de Población. Córdoba, Argentina, 24 al 26 de septiembre de 2008.

. "La emigración en Argentina: 1960-2000", in TORRADO, Susana (comp.). Población y bienestar en la Argentina del primero al segundo Centenario. Tomo I. Buenos Aires: Editorial Edhasa, 2007.

CELADE (Centro Latinoamericano y Caribeño de Demografía). Censos de población de 1990: selección de documentos del CELADE. (LC/DEM/G.77), serie A 193. Santiago de Chile: CEPAL, 1989.

${ }^{30}$ MARTÍNEZ PIZARRO, Jorge. América Latina migración internacional, derechos humanos y desarrollo. 
CEPAL-CELADE. Observatorio Demográfico 7 Proyección de población. Santiago de Chile, 2009.

MAGUID, Alicia. "La emigración internacional a través de los censos en países de origen: evaluación de resultados y recomendaciones", in Serie Población y Desarrollo, n. 86 (LC/L.2968-P). Santiago de Chile: CEPAL, 2008.

MARTÍNEZ PIZARRO, Jorge. (ed.). América Latina y el Caribe: migración internacional, derechos humanos y desarrollo. (LC/G.2358), Libros de la CEPAL 97. Santiago de Chile: CEPAL, 2008.

. Desafíos para mejorar la información sobre migración internacional: notas de discusión. Buenos Aires: Congreso Argentino sobre Migración y Asilo, 2006.

. "La migración internacional en los censos de población", in Revista Notas de Población, n. 69 (LC/G.2062/E). Santiago de Chile: CEPAL, 1999, p. 61-90.

- "Medición e información sobre la migración internacional a partir de los censos: lecciones, desafíos y oportunidades", in Revista Notas de Población, n. 88 (LC/G.2409/P). Santiago de Chile: CEPAL, 2009, p. 97-134.

PELLEGRINO, Adela. "Presentación del taller", in PELLEGRINO, Adela (comp.). Migración e integración. Nuevas formas de movilidad de la población. Montevideo: TRILCE, Universidad de la República, 1995, p. 11-23.

UNITED NATIONS. Principles and Recommendations for Population and Housing Censuses, Revision 2 (ST/ESA/STAT/SER.M/67/Rev.2), Statistical papers. New York: Department of Economic and Social Affairs, Statistics Division, 2008.

\section{Abstract}

\section{International migration in the 2010 census in Latin American and Caribbean countries}

This article discusses measurements of international migration in Latin American and Caribbean countries until 2010. It presents an overview based on the analysis of ten population censuses, which completed the survey phase by mid-2012. The censuses are described in relation to their strengths and limitations, and are compared with previous practices, international recommendations, and adopted modalities. The article also points out the emergence of new topics of interest, such as inquiries by emigrants.

Keywords: Measurement of international migration; Latin American and Caribbean countries; 2010 population censuses.

Recibido para publicación en 25/08/2012.

Aceptado para publicación en 02/10/2012.

Received for publication in August, 25 ${ }^{\text {th }}, 2012$. Accepted for publication in October, 02 ${ }^{\text {th }}, 2012$. 